\title{
Three-dimensional assessment of coronary high-intensity plaques with T1-weighted cardiovascular magnetic resonance imaging to predict periprocedural myocardial injury after elective percutaneous coronary intervention
}

Hayato Hosoda ${ }^{1,2}$, Yasuhide Asaumi ${ }^{1 *}$ (D), Teruo Noguchi ${ }^{1}$, Yoshiaki Morita ${ }^{3}$, Yu Kataoka ${ }^{1,2}$, Fumiyuki Otsuka', Kazuhiro Nakao ${ }^{1}$, Masashi Fujino ${ }^{1}$, Toshiyuki Nagai ${ }^{1}$, Michikazu Nakai ${ }^{4}$, Kunihiro Nishimura ${ }^{4}$, Atsushi Kono ${ }^{3}$, Yoshiaki Komori ${ }^{5}$, Tomoya Hoshi ${ }^{6}$, Akira Sato ${ }^{6}$, Tomohiro Kawasaki ${ }^{7}$, Chisato Izumi ${ }^{1}$, Kengo Kusano ${ }^{1}$, Tetsuya Fukuda ${ }^{3}$ and Satoshi Yasuda ${ }^{1,2}$

\begin{abstract}
Background: Periprocedural myocardial injury (pMI) is a common complication of elective percutaneous coronary intervention $(\mathrm{PCl})$ that reduces some of the beneficial effects of coronary revascularization and impacts the risk of cardiovascular events. We developed a 3-dimensional volumetric cardiovascular magnetic resonance (CMR) method to evaluate coronary high intensity plaques and investigated their association with $\mathrm{pMl}$ after elective $\mathrm{PCl}$.

Methods: Between October 2012 and October 2016, 141 patients with stable coronary artery disease underwent T1-weighted CMR imaging before PCI. A conventional 2-dimensional CMR plaque-to-myocardial signal intensity ratio (2D-PMR) and the newly developed 3-dimensional integral of PMR (3Di-PMR) were measured. 3Di-PMR was determined as the sum of PMRs above a threshold of $>1.0$ for voxels in a target plaque. pMI was defined as highsensitivity cardiac troponin $\mathrm{T}>0.07 \mathrm{ng} / \mathrm{mL}$.

Results: pMl following PCI was observed in 46 patients (33\%). 3Di-PMR was significantly higher in patients with $\mathrm{pMI}$ than those without pMI. The optimal 3Di-PMR cutoff value for predicting pMI was 51 PMR $^{*} \mathrm{~mm}^{3}$ and the area under the receiver operating characteristic curve $(0.753)$ was significantly greater than that for 2D-PMR $(0.683, P=$ 0.015). 3Di-PMR was positively correlated with lipid volume $(r=0.449, P<0.001)$ based on intravascular ultrasound. Stepwise multivariable analysis showed that $3 \mathrm{Di}-\mathrm{PMR} \geq 51 \mathrm{PMR}^{*} \mathrm{~mm}^{3}$ and the presence of a side branch at the PCl target lesion site were significant predictors of pMl (odds ratio [OR], 11.9; 95\% confidence interval [Cl], 4.6-30.4, $P<$ 0.001 ; and $\mathrm{OR}, 4.14 ; 95 \% \mathrm{Cl}, 1.6-11.1, P=0.005$, respectively).
\end{abstract}

Conclusions: 3Di-PMR coronary assessment facilitates risk stratification for pMl after elective PCl.

Trial registration: retrospectively registered.

Keywords: Magnetic resonance imaging, Coronary atherosclerosis, Percutaneous coronary intervention

\footnotetext{
*Correspondence: asaumiya@ncvc.go.jp

'Department of Cardiovascular Medicine, National Cerebral and

Cardiovascular Center, 6-1 Kishibe-Shimmachi, Suita 564-8565, Japan

Full list of author information is available at the end of the article
}

(c) The Author(s). 2020 Open Access This article is distributed under the terms of the Creative Commons Attribution 4.0 International License (http://creativecommons.org/licenses/by/4.0/), which permits unrestricted use, distribution, and reproduction in any medium, provided you give appropriate credit to the original author(s) and the source, provide a link to the Creative Commons license, and indicate if changes were made. The Creative Commons Public Domain Dedication waiver (http://creativecommons.org/publicdomain/zero/1.0/) applies to the data made available in this article, unless otherwise stated. 


\section{Introduction}

Percutaneous coronary intervention (PCI) has become the most common procedure for coronary revascularization in patients with both stable and unstable coronary artery disease (CAD). Although technical advances in PCI have resulted in a safer therapeutic procedure, 5$30 \%$ of patients undergoing elective PCI demonstrate evidence of periprocedural myocardial injury (pMI) from the procedure itself $[1,2]$. The extent of pMI as determined by evaluation of creatine phosphokinase MB isoenzyme, high-sensitivity troponin $\mathrm{T}$ (hs-cTnT), and troponin I is significant enough to be prognostically important $[1,3]$. Therefore, pMI might reduce some of the beneficial effects of coronary revascularization.

Coronary high-intensity plaques (HIPs) with a high plaque-to-myocardial signal intensity ratio (PMR) on noncontrast T1-weighted (T1w) cardiovascular magnetic resonance (CMR) imaging are associated with future coronary events and PCI-related pMI [4-7]. However, current HIP-PMR evaluation is solely based on signal intensity without consideration of plaque volume, which yields a 2dimensional PMR (2D-PMR) based on a coronary plaque as a region of interest (ROI) $[4,5]$. Since larger plaque volume is associated with cardiovascular events such as acute myocardial infarction, which includes pMI $[8,9]$, more accurate, quantitative assessment is needed to predict cardiovascular events.

Herein, we sought to examine whether 3-dimensional integral (3Di) volumetric assessment of coronary HIPs on non-contrast $\mathrm{T} 1 \mathrm{w}$ imaging would have better predictive value for pMI after elective PCI compared with the current 2D-PMR in patients with stable CAD.

\section{Methods}

\section{Study patients}

From October 2012 to October 2016, 215 consecutive stable CAD patients undergoing elective PCI following noncontrast T1w imaging for coronary atherosclerosis were included in this study retrospectively. CMR was used to evaluate the characteristics of the target coronary plaque. We excluded 74 patients who had lesions involving the left main trunk $(n=12)$, chronic total occlusion $(n=17)$, previous cardiac surgery $(n=1)$, renal insufficiency (serum creatinine $\geq 1.8 \mathrm{mg} / \mathrm{dL}, \mathrm{n}=1)$, multi-segment PCI $(n=2)$, missing baseline hs-cTnT data $(n=21)$, poor CMR image quality $(n=8)$, or rotational or direct coronary atherectomy during PCI $(\mathrm{n}=12)$. Ultimately, 141 patients $(122$ males, $87 \%)$ with a median age of 68 years (interquartile range [IQR], 60-73 years) were analyzed in this study. This study was approved by our institutional review board (M26-037-2).

\section{CMR coronary plaque imaging}

Non-contrast T1w imaging was performed at $3 \mathrm{~T}$ (MAGNETOM Verio; Siemens AG Healthcare Sector, Erlangen,
Germany) with a 32-channel cardiac coil. The procedures used to acquire CMR images in this study have been previously described $[6,10,11]$. Briefly, coronary plaque imaging was performed using an inversion recoveryprepared 3D T1w turbo fast low-angle shot sequence with an electrocardiographic (ECG) trigger, navigator-gated free-breathing, and fat suppression. Transaxial sections covered the entire heart (inversion time, $650 \mathrm{~ms}$; field of view, $280 \mathrm{~mm} \times 228 \mathrm{~mm}$; acquisition matrix, $256 \times 187$; reconstruction matrix, $512 \times 374$; acquisition slice thickness, $1.0 \mathrm{~mm}$; reconstruction spatial resolution, $0.55 \mathrm{~mm} \times 0.60$ $\mathrm{mm} \times 1.0 \mathrm{~mm}$; repetition time/echo, $4.7 \mathrm{~ms} / 2.13 \mathrm{~ms}$; flip angle, $12^{\circ}$; GRAPPA factor, 2 ; navigator gating window, \pm $1.5-2.5 \mathrm{~mm}$; and data acquisition window, 84-120 ms). The average navigator efficiency was $35.7 \pm 8.2 \%$. To identify the coronary segments of plaques evaluated on $\mathrm{T} 1 \mathrm{w}$ images in patients who did not undergo computed tomography angiography (CTA), 3D coronary CMR angiograms using a free-breathing, navigator-gated, ECG-triggered, fat-saturated, segmented gradient echo sequences were obtained in the axial plane to cover the entire heart (repetition time, $3.27 \mathrm{~ms}$; echo time, $1.35 \mathrm{~ms}$; flip angle, $12^{\circ}$; field of view, $280 \times 227$; acquisition matrix, $256 \times 175$; reconstruction matrix, $512 \times 350$, acquisition slice thickness 1.0 $\mathrm{mm}$; acquisition special resolution, $1.30 \mathrm{~mm} \times 1.09 \mathrm{~mm} \times$ $1.56 \mathrm{~mm}$; reconstruction spatial resolution, $0.55 \mathrm{~mm} \times 0.60$ $\mathrm{mm} \times 1.0 \mathrm{~mm}$; partial Fourier, 6/8; data window duration, $98 \mathrm{~ms}$ ). Trigger delay and acquisition windows were based on the duration of minimal right coronary artery motion as determined on cine-CMR imaging. The mean acquisition times for plaque imaging and coronary CMR angiography were $23.1 \pm 3.8 \mathrm{~min}$ and $17.4 \pm 2.4 \mathrm{~min}$, respectively.

\section{Three-dimensional plaque analysis with CMR}

On CMR analysis, the coronary vasculature tree was subdivided into 8 segments [12]. In brief, the right coronary artery was analyzed in 3 segments (segments 1, 2, 3 ). The left coronary artery was analyzed in 4 segments that comprised the left anterior descending artery (segments 6, 7) and left circumflex artery (segments 11, 13). Since lesions in the left main trunk, both in the left anterior descending artery and left circumflex artery (segment 5), are associated with a large ischemic burden, PCI for left main stenosis is indicated only in patients with a low SYNTAX score and non-multivessel disease [13], and PCI for left main lesions may be susceptible to operator bias. Therefore we excluded left main lesions in the present analysis. For segment identification, segments were pre-defined according to the distance from the vessel's origin $[5,6,12]$. The location of a coronary plaque was determined by carefully comparing either CTA or CMR angiogram or invasive coronary arteriography images during PCI using fiduciary points (e.g. side branches and vessel bends). Once a coronary plaque had 
been confirmed with either CTA or coronary CMR angiogram, the corresponding areas on coronary $\mathrm{T} 1 \mathrm{w}$ images were carefully matched using the surrounding cardiac and chest wall structures [11]. Fifty-seven patients underwent coronary CTA and co-registration between coronary T1w imaging and CTA (Additional file 1: Supplemental Methods and Table S1).

2D-PMR was assessed as previously described $[4-6,11]$. For 3D analysis of coronary plaques on non-contrast $\mathrm{T} 1 \mathrm{w}$ imaging, OsiriX MD software (version 8.0.2, Pixmeo, Geneva, Switzerland) was used. The 3D integral of the plaqueto-myocardium signal intensity ratio (3Di-PMR) was defined as the integral of voxel volume $(0.55 \mathrm{~mm} \times 0.60 \mathrm{~mm} \times 1.0$ $\mathrm{mm}=0.33 \mathrm{~mm}^{3}$ ) multiplied by its PMR value $>1.0$ from a coronary plaque (Fig. 1, Additional file 1: Figure S1, Figure S2, Figure S3, and Additional file 2: Step 1, Videos S1, Additional file 3: Step 2, Videos S2, Additional file 4: Step 3, Videos S3). The algorithm for plaque segmentation was based on a region-growing technique, a function built into the OsiriX MD software consisting of the following steps (Figure 2 and Additional file 2: Step 1, Videos S1, Additional file 3: Step 2, Videos S2, Additional file 4: Step 3, Videos S3) [14]. First, an elliptical ROI of $\geq 1.0 \mathrm{~cm}^{2}$ in area was drawn around the myocardium near a coronary plaque. The mean signal intensity of the ROI was defined as the lower threshold of the signal intensity of the coronary plaque. Next, entire voxels of a coronary plaque above the threshold PMR of 1.0 were segmented automatically within contiguous slices to calculate both the integral signal intensity and voxel volume (3D region growing: Step1, Additional file 1: Figure S1 and Additional file 2: Step 1, Video S1) [14]. Segments with a PMR above the threshold that were not analyzed by the automatic 3D segmentation, which was defined as those with expansion of the ROI beyond the boundaries of the vessel, were divided into 2 groups according to the course of the coronary vessel: those running along axial slices (Step 2, segments 1, 3, 6, 7) and those running perpendicular to axial slices (Step 3, segments 2, 11, 13). These segments were then analyzed using the methods described below.

Since the mean diameter of proximal coronary segments in Japanese subjects is $4-5 \mathrm{~mm}$ [15], we used a slice thickness of $1 \mathrm{~mm}$ to cover the entirety of each vessel. Therefore, in the Step 2 method (for segments 1, 3, 6, 7), 3Di-PMR was determined by performing automatic contiguous $2 \mathrm{D}$ segmentation of each coronary plaque above the PMR threshold, in not more than 5 contiguous slices and without expansion beyond the boundaries of the vessel (Additional file 1: Figure S2-a, S2-b and Additional file 3: Step 2, Video S2).

For segments 2, 11, 13, the lower segmentation threshold was increased from the signal intensity of the nearby myocardium in increments of 5 units until there was no longer expansion beyond the vessel boundaries (Additional file 1: Figure S3 and Additional file 4: Step 3, Video 3).

\section{$\mathrm{PCl}$}

PCI was performed as previously reported [16]. pMI after elective PCI was defined as an increase in serum hs-cTnT levels to more than 5 times the upper limit of normal $(0.07 \mathrm{ng} /$

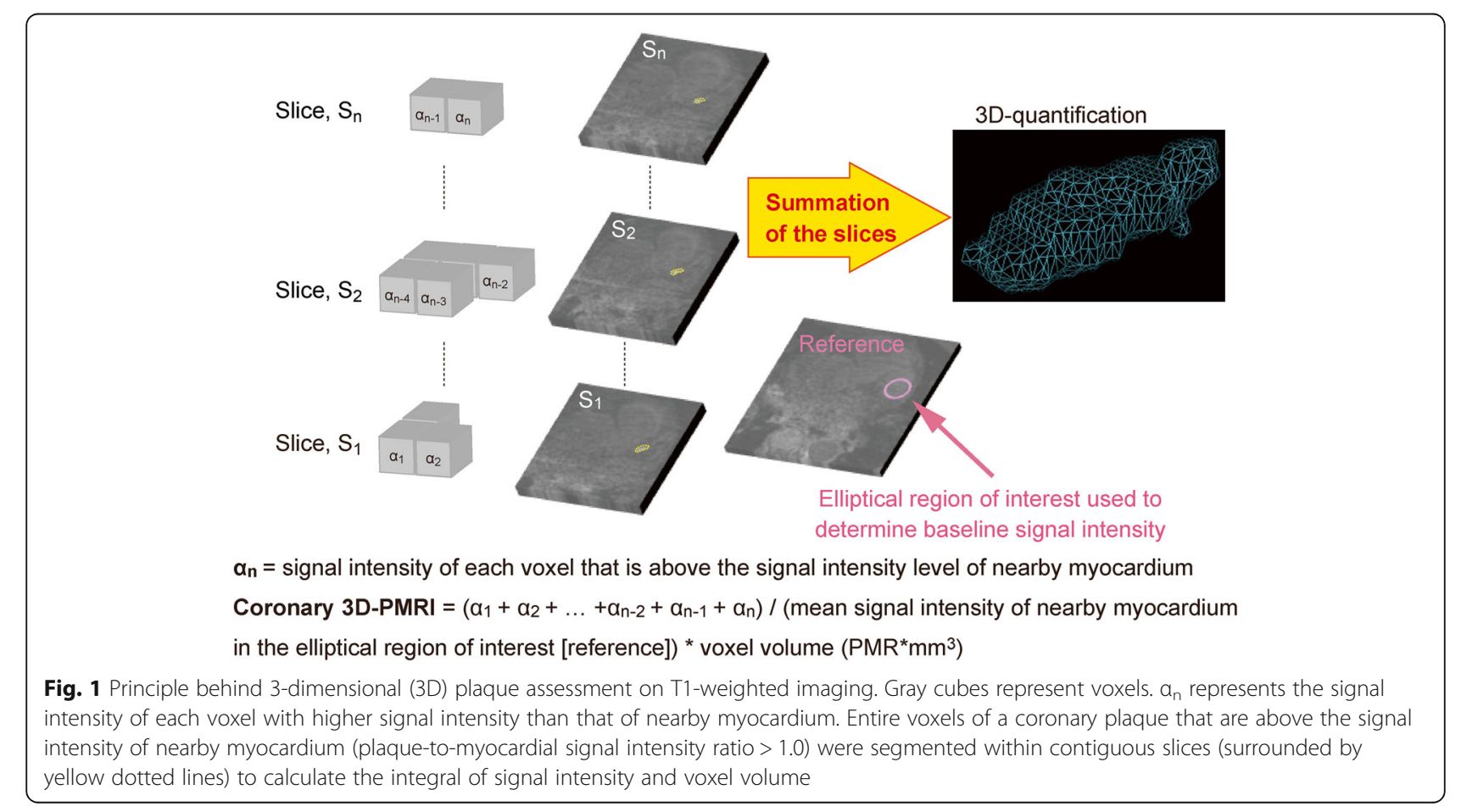




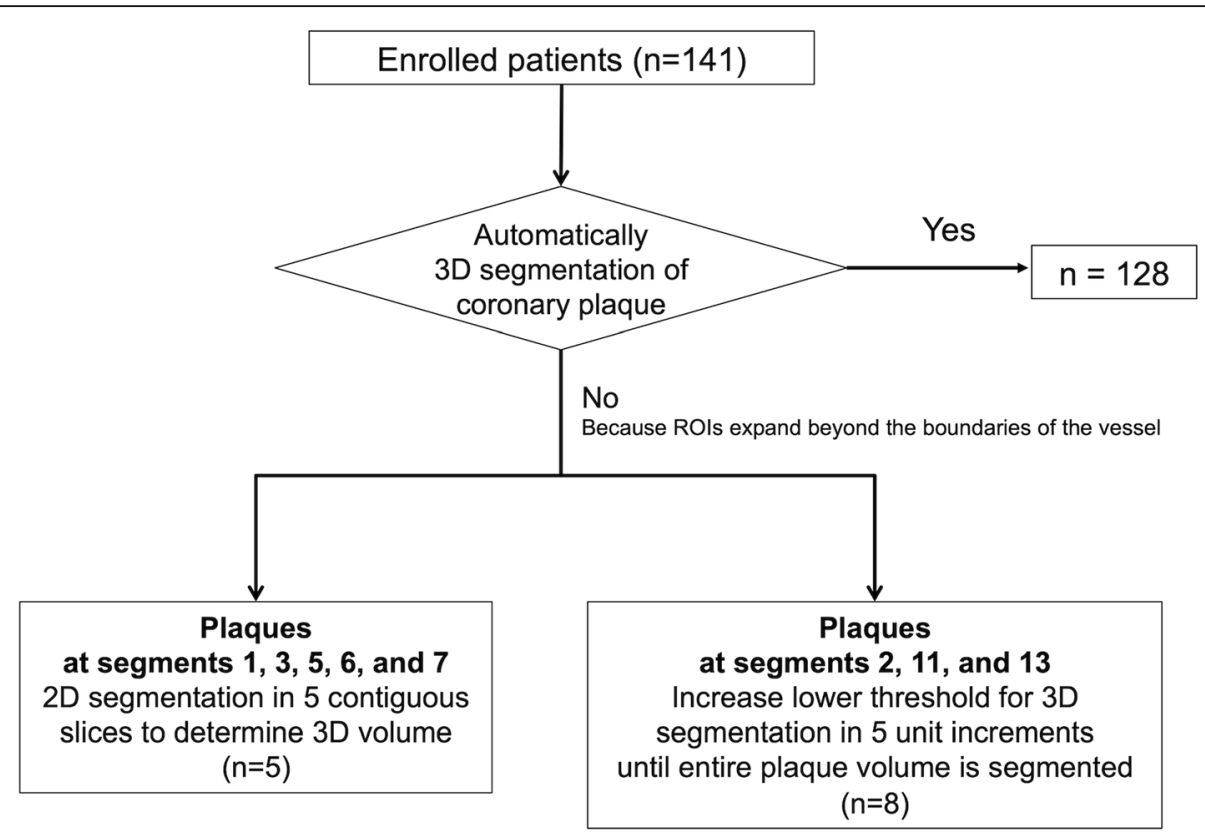

Fig. 2 Flow chart for 3D plaque assessment on T1-weighted imaging

$\mathrm{mL}$ ) at $24 \mathrm{~h}$ after PCI. Slow flow was defined as the Thrombolysis In Myocardial Infarction (TIMI) grade 0, 1, or 2 flow in the distal infarct-related artery despite the absence of occlusion or dissection at the treatment site. The presence of a side branch was defined as the presence of a vessel of $\geq 1.5 \mathrm{~mm}$ in diameter within the target lesion. Side branch occlusion was defined as TIMI grade 0,1 , or 2 flow during PCI.

\section{Intravascular ultrasound image analysis}

Intravascular ultrasound (IVUS) images were obtained during PCI and analyzed in 126 patients. IVUS was performed using a commercially available IVUS catheter (View It; Terumo, Tokyo, Japan) with $0.5 \mathrm{~mm} / \mathrm{sec}$ autopullback. Quantitative and qualitative analysis were performed in a blinded manner as previously described $[4,6$, 7]. Positive remodeling was defined as a remodeling index of $>1.05$. Ultrasound attenuation was defined as IVUS images with backward signal attenuation of $\geq 180^{\circ}$ behind the plaque without dense calcium. The presence of ultrasound attenuation was defined as longitudinal attenuation length of $\geq 5 \mathrm{~mm}$ [17]. For tissue characterization, IVUS data were analyzed using the manufacturer's default setting on the basis of previous data used to define a range of integrated backscatter values. Coronary plaques were classified into three categories: lipid pool (blue), fibrosis (green/ yellow), and calcification (red) [9].

\section{Statistical analysis}

Values are presented as medians (IQR). Values were compared using the Mann-Whitney U test or Kruskal-Wallis test for multiple comparisons followed by the Steel-Dwass test for post hoc analysis. Categorical baseline variables were compared using Fisher's exact test or the chisquared test as appropriate. 3Di-PMR and 2D-PMR cutoff values for the development of coronary events were determined with receiver operating characteristic (ROC) analysis. To identify risk factors for pMI, univariable and multivariable logistic regression models were constructed using 3Di-PMR, attenuation, positive remodeling, calcification, type B2/C lesion, and presence of a side branch. Stepwise multivariable logistic regression with a $P$ value of 0.10 for backward elimination was performed to select the best predictive model. All analyses were conducted using JMP (version 12, SAS Japan, Tokyo, Japan) and Stata, version 14 (StataCorp LP, College Station, Texas, USA). A $P$ value less than 0.05 was considered statistically significant.

\section{Results}

Table 1 summarizes the baseline characteristics of the study patients. Of 141 patients, pMI was observed in 46 patients (33\%). Both 3Di-PMR and 2D-PMR were significantly higher in patients with pMI than those without pMI $(P<$ $0.001)$, although age, gender, coronary risk factors, medications, and pre-PCI hs-cTnT level were similar. As shown in Additional file 1: Figure S4-C, 3Di-PMR is strongly and positively correlated with 2D-PMR $(\mathrm{r}=0.832, P<0.001)$. Intraclass correlation coefficients with $95 \%$ confidence intervals (CIs) were calculated to assess intra- and interreader agreement regarding 3Di-PMR. The interval between initial analysis of 3Di-PMR of HIPs and secondary analysis was 3 months. The intra- and inter-reader intraclass correlation coefficients for 3Di-PMR were 0.947 (95\% 
Table 1 Baseline Characteristics of the Study Patients

\begin{tabular}{|c|c|c|c|}
\hline & No pMl $(n=95)$ & $\mathrm{pMI}(n=46)$ & $P$ Value \\
\hline Age, yrs & $68(61-74)$ & $67(59-73)$ & 0.582 \\
\hline Male & $83(87)$ & $39(85)$ & 0.793 \\
\hline $\mathrm{BMl}, \mathrm{kg} / \mathrm{m}^{2}$ & $25(22-27)$ & $24(22-27)$ & 0.514 \\
\hline Hypertension & $76(80)$ & $35(76)$ & 0.662 \\
\hline Hyperlipidemia & $86(91)$ & $42(91)$ & 1.000 \\
\hline Diabetes mellitus & $29(31)$ & $12(26)$ & 0.627 \\
\hline Current smoker & $11(12)$ & $7(15)$ & 0.594 \\
\hline Total cholesterol, mg/dL & 149 (134-173) & $160(144-177)$ & 0.338 \\
\hline LDL cholesterol, mg/dL & $82(67-102)$ & $82(71-100)$ & 0.811 \\
\hline HDL cholesterol, mg/dL & $44(38-52)$ & $46(38-55)$ & 0.273 \\
\hline Triglycerides, mg/dL & $135(97-192)$ & $129(94-167)$ & 0.356 \\
\hline Hemoglobin $A_{1 c}, \%$ & $6.0(5.6-6.3)$ & $5.9(5.7-6.2)$ & 0.378 \\
\hline Serum creatinine, mg/dL & $0.90(0.79-1.05)$ & $0.93(0.81-1.06)$ & 0.633 \\
\hline Multivessel disease & $28(29)$ & $18(39)$ & 0.258 \\
\hline Previous Ml & $14(15)$ & $10(22)$ & 0.342 \\
\hline LVEF, \% & $60(55-65)$ & $60(57-65)$ & 0.732 \\
\hline hs-cTnT before $\mathrm{PCl}, \mathrm{ng} / \mathrm{mL}$ & $\begin{array}{l}0.008 \\
(0.006-0.012)\end{array}$ & $\begin{array}{l}0.009 \\
(0.006-0.016)\end{array}$ & 0.543 \\
\hline hs-cTnT after PCl, ng/mL & $\begin{array}{l}0.027 \\
(0.020-0.042)\end{array}$ & $\begin{array}{l}0.138 \\
(0.094-0.276)\end{array}$ & $<0.001$ \\
\hline \multicolumn{4}{|l|}{ Medications } \\
\hline Statin & $88(93)$ & $41(89)$ & 0.527 \\
\hline Beta-blocker & $67(71)$ & $27(59)$ & 0.185 \\
\hline ACE inhibitor or ARB & $50(53)$ & $24(52)$ & 1.000 \\
\hline \multicolumn{4}{|l|}{ MRI findings } \\
\hline 2D-PMR & $1.15(0.98-1.36)$ & $1.66(1.09-2.93)$ & $<0.001$ \\
\hline 3Di-PMR, PMR* $\mathrm{mm}^{3}$ & $9(0-49.8)$ & $88.4(32.2-203.7)$ & $<0.001$ \\
\hline \multicolumn{4}{|l|}{ Angiographic findings and $\mathrm{PCl}$ parameters } \\
\hline Mean reference vessel diameter $(\mathrm{mm})$ & $3.50(3.06-4.19)$ & $3.45(3.07-4.24)$ & 0.852 \\
\hline Ratio of the maximal balloon diameter to the vessel diameter & $0.95(0.79-1.06)$ & $0.96(0.83-1.06)$ & 0.852 \\
\hline Total lesion length (mm) & $16(10-26)$ & $18(12-28)$ & 0.439 \\
\hline
\end{tabular}

Values are medians (interquartile range) or $\mathrm{n}(\%)$

2D-PMR 2-dimensional plaque-to-myocardium signal intensity ratio, 3Di-PMR 3-dimensional integral of the plaque-to-myocardium signal intensity ratio, $A C E$ Angiotensin-converting enzyme, ARB Angiotensin II receptor blocker, BMI Body mass index, HDL High-density lipoprotein, $h s-c T n T$ High-sensitivity cardiac troponin T, LDL Low-density lipoprotein, LVEF Left ventricular ejection fraction, MI Myocardial infarction, MRI Magnetic resonance imaging, $P C I$ Percutaneous coronary intervention, $p M I$ Periprocedural myocardial injury, $P M R^{*} \mathrm{~mm}^{3}$ the unit of 3Di-PMR; defined as the integral of voxel volume multiplied by its PMR value > 1.0 from a coronary plaque

CI, 0.911-0.969) and 0.926 (95\% CI, 0.876-0.957), respectively. All correlation coefficients for calculating 3Di-PMR were greater than 0.8 , indicating good intra- and interobserver agreement.

On the basis of the ROC analysis, a 3Di-PMR value of 51 $\mathrm{PMR}^{*} \mathrm{~mm}^{3}$ was identified as the best cutoff for predicting pMI after elective PCI, with an area under the curve (AUC) of 0.753 (95\% CI, 0.665-0.841). At this value, the sensitivity and specificity for predicting pMI were 74 and $76 \%$, respectively. Table 2 and Additional file 1: Figure S4-D and S4-E show the lesion characteristics and incidence of pMI and slow flow phenomenon by 3Di-PMR cutoff value. Patients with $3 \mathrm{Di}-\mathrm{PMR} \geq 51 \mathrm{PMR}^{*} \mathrm{~mm}^{3}$ had a larger plaque volume, longer attenuation length, higher remodeling index, larger lipid volume, and higher incidence of both pMI and slow flow phenomenon than those with 3Di$\mathrm{PMR}<51 \mathrm{PMR}^{*} \mathrm{~mm}^{3}$. To show the clinical significance of 3Di-PMR, previously described predictors of pMI were compared with 3Di-PMR. From ROC analysis (Table 3), 3Di-PMR had a significantly higher AUC (0.753) than 2DPMR (0.683 [95\%CI, 0.609-0.782]) on T1w imaging ( $P=$ $0.015)$, as well as other IVUS or coronary angiography 
Table 2 Lesion Characteristics Stratified by 3Di-PMR Cutoff

\begin{tabular}{|c|c|c|c|}
\hline & 3Di-PMR < $51 \mathrm{PMR}^{*} \mathrm{~mm}^{3}(n=84)$ & 3 Di-PMR $\geq 51$ PMR $^{*} \mathrm{~mm}^{3}(n=57)$ & $P$ Value \\
\hline \multicolumn{4}{|l|}{ Angiographic findings } \\
\hline Target vessel & & & 0.375 \\
\hline $\mathrm{LAD} / \mathrm{LCX} / \mathrm{RCA}$ & $52 / 14 / 18$ & 29/9/19 & \\
\hline Type B2/C lesion & $46(55)$ & $35(61)$ & 0.434 \\
\hline Side branch at the $\mathrm{PCl}$ target lesion site & $53(63)$ & $33(58)$ & 0.534 \\
\hline Calcification & $17(20)$ & $7(12)$ & 0.217 \\
\hline Total lesion length, mm & $17(10-26)$ & $18(11-28)$ & 0.769 \\
\hline \multicolumn{4}{|l|}{$\mathrm{PCl}$ parameters } \\
\hline Stent diameter, mm & $3.0(2.8-3.3)$ & $3.25(3.0-3.5)$ & $<0.001$ \\
\hline Stent length, mm & $24(18-33)$ & $24(18-37)$ & 0.500 \\
\hline Post-dilatation balloon size, $\mathrm{mm}$ & $3.3(3.0-3.5)$ & $3.5(3.3-4.0)$ & $<0.001$ \\
\hline Cross-sectional IVUS parameters & $(n=74)$ & $(n=52)$ & \\
\hline \multicolumn{4}{|l|}{ Grayscale IVUS analysis } \\
\hline Lesion EEM CSA, mm² & $8.4(6.1-11.3)$ & $12.9(8.9-15.8)$ & $<0.001$ \\
\hline Lesion lumen CSA, mm² & $1.3(1.0-2.0)$ & $1.3(0.9-2.0)$ & 0.560 \\
\hline Lesion $\mathrm{P}+\mathrm{M}$ CSA, $\mathrm{mm}^{2}$ & $6.6(5.0-9.4)$ & $11.3(8.1-13.5)$ & $<0.001$ \\
\hline Plaque burden, \% & $83(77-86)$ & $89(85-92)$ & $<0.001$ \\
\hline Remodeling index & $0.89(0.78-1.05)$ & $1.06(0.91-1.19)$ & $<0.001$ \\
\hline Positive remodeling & $19(26)$ & $26(50)$ & 0.004 \\
\hline Ultrasound attenuation & $6(8)$ & $18(35)$ & $<0.001$ \\
\hline Attenuation length, mm & $0(0-1.7)$ & $3.6(0-6.6)$ & $<0.001$ \\
\hline Intracoronary thrombus & $2(3)$ & $9(18)$ & 0.015 \\
\hline \multicolumn{4}{|l|}{ IB-IVUS parameters } \\
\hline Lipid area, \% & $68(57-80)$ & $81(68-86)$ & 0.003 \\
\hline Fibrous area, \% & $24(17-38)$ & $17(13-28)$ & 0.007 \\
\hline Calcified area, \% & $3.7(1.1-7.4)$ & $2.0(0.4-3.7)$ & 0.030 \\
\hline \multicolumn{4}{|l|}{ Volumetric IVUS analysis } \\
\hline \multicolumn{4}{|l|}{ Grayscale IVUS parameters } \\
\hline EEM volume, $\mathrm{mm}^{3}$ & $174(135-247)$ & $246(171-336)$ & $<0.001$ \\
\hline Lumen volume, $\mathrm{mm}^{3}$ & $62(47-93)$ & $75(57-107)$ & 0.147 \\
\hline Total plaque volume, $\mathrm{mm}^{3}$ & $104(82-140)$ & $158(114-210)$ & $<0.001$ \\
\hline Plaque burden, $\%$ & $61(55-67)$ & $66(62-74)$ & $<0.001$ \\
\hline \multicolumn{4}{|l|}{ IB-IVUS parameters } \\
\hline Lipid volume, \% & $68(55-78)$ & $71(63-83)$ & 0.039 \\
\hline Fibrous volume, \% & $28(20-40)$ & $25(16-33)$ & 0.052 \\
\hline Calcified volume, \% & $3.5(1.6-7.3)$ & $2.4(0.9-4.0)$ & 0.039 \\
\hline
\end{tabular}

Values are medians (interquartile range) or $\mathrm{n}(\%)$

2D-PMR 2-dimensional plaque-to-myocardium signal intensity ratio, 3Di-PMR 3-dimensional integral of the plaque-to-myocardium signal intensity ratio, CSA Crosssectional area, EEM External elastic membrane, IB-IVUS Integrated backscatter intravascular ultrasound, LAD Left anterior descending coronary artery, LCX Left circumflex coronary artery, $P+M$ Plaque plus media, $P C I$ Percutaneous coronary intervention, $P M R^{*} m m^{3}$ the unit of $3 D i-P M R$; the integral of voxel volume multiplied by its PMR value $>1.0$ from a coronary plaque, RCA Right coronary artery

derived indices; attenuation length $(0.641$ [95\% CI, 0.5390.477], $P=0.038)$, remodeling index $(0.547$ [95\% CI, 0.4560.637], $P=0.013)$, and prevalence of type $\mathrm{B} 2 / \mathrm{C}$ lesion (0.525 [95\% CI, 0.438-0.613], $P<0.001)$, bifurcation lesion (0.612 [95\% CI, 0.532-0.692], $P=0.039$ ), and calcification
(0.503 [95\% CI, 0.436-0.570], $P<0.001)$. Figure 3 shows the correlation between 3Di-PMR and IVUS derived indices. 3Di-PMR was significantly positively correlated with total plaque volume and lipid volume $(\mathrm{r}=0.449, P<0.001$ and $\mathrm{r}=0.426, P<0.001$, respectively). 
Table 3 Receiver Operating Characteristic Analysis Demonstrating the Prediction of Periprocedural Myocardial Injury

\begin{tabular}{lll}
\hline Variable & AUC $(95 \% \mathrm{Cl})$ & $P$ Value \\
\hline 3Di-PMR, PMR* $\mathrm{mm}^{3}$ & $0.753(0.665-0.841)$ & Referent \\
2D-PMR & $0.683(0.609-0.782)$ & 0.015 \\
Attenuation length & $0.641(0.539-0.744)$ & 0.038 \\
Remodeling index & $0.547(0.456-0.637)$ & 0.013 \\
Side branch & $0.612(0.532-0.692)$ & 0.039 \\
Type B2/C lesion & $0.525(0.438-0.613)$ & $<0.001$ \\
Calcification & $0.503(0.436-0.570)$ & $<0.001$ \\
\hline
\end{tabular}

3Di-PMR 3-dimensional integral of the plaque-to-myocardium signal intensity ratio, AUC Area under the curve, $\mathrm{Cl}$ Confidence interval, $P M R^{*} \mathrm{~mm}^{3}$ the unit of 3Di-PMR; the integral of voxel volume mulplied by its PMR value $>1.0$ from a coronary artery

Because our previous study had demonstrated that a 2D-PMR cutoff value of 1.4 is a significant predictor of coronary events [5], we subdivided the study patients into the following 4 groups according to the 3Di-PMR cutoff value of $51 \mathrm{PMR}^{*} \mathrm{~mm}^{3}$ and the 2D-PMR cutoff value of 1.4: 2D-PMR $<1.4+3 \mathrm{Di}-\mathrm{PMR}<51 \mathrm{PMR}^{*} \mathrm{~mm}^{3}\left(2 \mathrm{D}^{\text {low }} 3-\right.$ $\mathrm{D}^{\text {low }}$ group: $\left.n=78\right), \quad 2 \mathrm{D}-\mathrm{PMR} \geq 1.4+3 \mathrm{Di}-\mathrm{PMR}<51$ PMR $* \mathrm{~mm}^{3}\left(2 \mathrm{D}^{\text {high }} 3 \mathrm{D}^{\text {low }}\right.$ group: $\left.n=6\right), 2 \mathrm{D}-\mathrm{PMR}<1.4+$ $3 \mathrm{Di}-\mathrm{PMR} \geq 51 \mathrm{PMR}^{*} \mathrm{~mm}^{3}{ }^{3}$ (2D ${ }^{\text {low }} 3 \mathrm{D}^{\text {high }}$ group: $\left.n=15\right)$, and $2 \mathrm{D}-\mathrm{PMR} \geq 1.4+3 \mathrm{Di}-\mathrm{PMR} \geq 51 \quad \mathrm{PMR}^{*} \mathrm{~mm}^{3} \quad\left(2 \mathrm{D}^{\text {high }} 3 \mathrm{D}^{\text {high }}\right.$ group: $n=42$ ). Figure 4 shows representative $2 \mathrm{D}$ and $3 \mathrm{D}$ plaque assessments on T1w imaging. Coronary plaques with $2 \mathrm{D}-\mathrm{PMR}<1.4+3 \mathrm{Di}-\mathrm{PMR} \geq 51 \quad \mathrm{PMR}^{*} \mathrm{~mm}^{3}$ in the proximal right coronary artery (a patient with $2 \mathrm{D}^{\text {low }} 3 \mathrm{D}^{\text {high }}$ plaque; Patient A: panels a-e), 2D-PMR $\geq 1.4+3$ Di-PMR $<$ $51 \mathrm{PMR}^{*} \mathrm{~mm}^{3}$ in the proximal left anterior descending artery (a patient with $2 \mathrm{D}^{\text {high }} 3 \mathrm{D}^{\text {low }}$ plaque; Patient B: panels $\mathrm{f}-\mathrm{j}$ ), and $2 \mathrm{D}-\mathrm{PMR} \geq 1.4+3 \mathrm{Di}-\mathrm{PMR} \geq 51 \mathrm{PMR}^{*} \mathrm{~mm}^{3}$ in the proximal left anterior descending artery (a patient with $2 \mathrm{D}^{\text {high }} 3 \mathrm{D}^{\text {high }}$ plaque; Patient C: panels $\mathrm{k}-\mathrm{o}$ ) and corresponding CTA images are shown. Coronary CTA $(a, f, k)$, and axial images (b, g, l), sagittal images (c, h, m), color maps (d, I, n), and volume images (3D plaque: e, j, n) and non-contrast $\mathrm{T} 1 \mathrm{w}$ images are shown in Figure 4. Among the four groups based on 2D-PMR and 3Di-PMR cutoff values, the incidence of $\mathrm{pMI}$ was highest in the $2 \mathrm{D}^{\text {high }} 3 \mathrm{D}^{\text {high }}$ group (64.3\%), and lowest in the $2 \mathrm{D}^{\text {high }} 3 \mathrm{D}^{\text {low }}$ group (0\%) $(P<0.001$, Figure 5). Of note, even in patients with 2D$\mathrm{PMR}<1.4$, the incidence of pMI among those with 3Di$\mathrm{PMR} \geq 51$ PMR* $\mathrm{mm}^{3}$ was significantly higher than in patients with $3 \mathrm{Di}-\mathrm{PMR}<51 \mathrm{PMR}^{*} \mathrm{~mm}^{3}\left(2 \mathrm{D}^{\text {low }} 3 \mathrm{D}^{\text {high }}\right.$ group: $46.7 \%$ vs. $2 \mathrm{D}^{\text {low }} 3 \mathrm{D}^{\text {low }}$ group: $15.4 \%, P=0.006$; Figure 5 ).

As shown in Table 4, univariable Cox regression analysis showed that $3 \mathrm{Di}-\mathrm{PMR} \geq 51 \mathrm{PMR} * \mathrm{~mm}^{3}$, attenuation detected on IVUS, and the presence of a side branch at the PCI target lesion site were associated with pMI. Multivariable analysis showed that $3 \mathrm{Di}-\mathrm{PMR} \geq 51 \mathrm{PMR}^{*} \mathrm{~mm}^{3}$ and the presence of a side branch at the PCI target lesion site

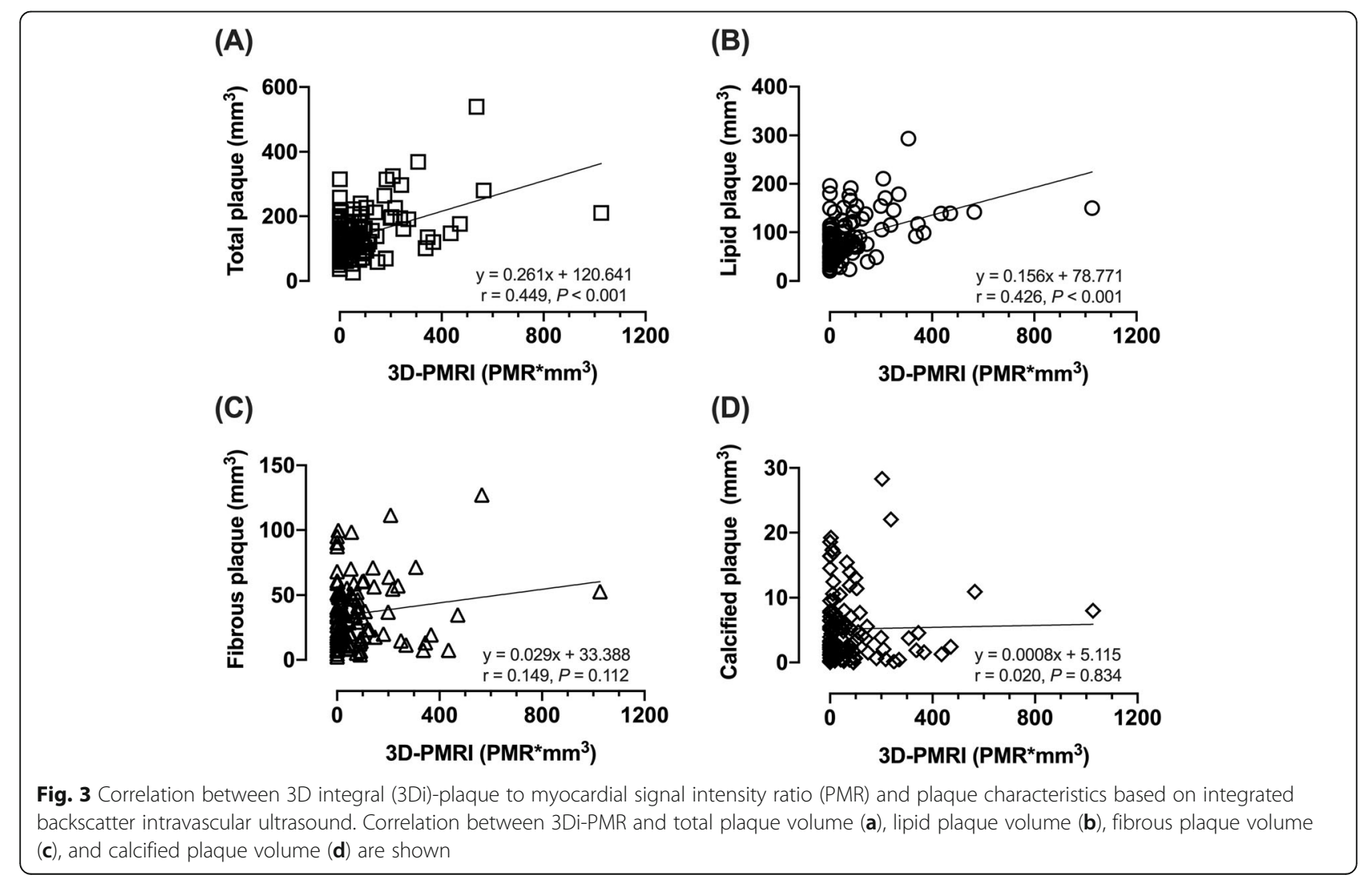




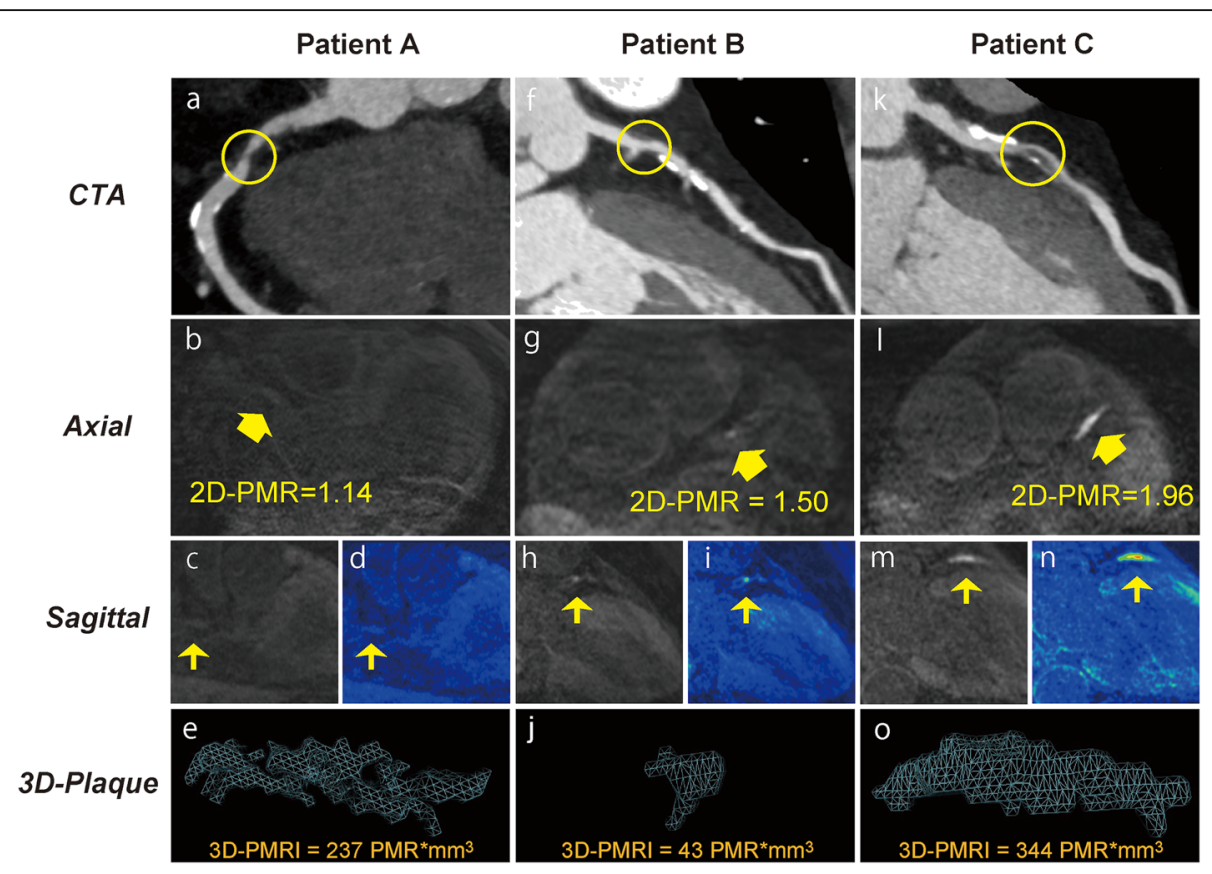

Fig. 4 Representative 2-dimensional and 3-dimensional plaque assessment on T1-weighted imaging. Coronary plaques with $2 D^{\text {low }} 3 D^{\text {high }}$ in the proximal right coronary artery (2D-PMR, 1.14; 3Di-PMR, $237 \mathrm{PMR}^{*} \mathrm{~mm}^{3}$; Patient $\mathrm{A}$ : a-e), $2 \mathrm{D}^{\text {high }} 3 \mathrm{D}^{\mathrm{low}}$ in the proximal left anterior descending artery (LAD) (2D-PMR, 1.50; 3Di-PMR, 43 PMR* $^{*} m^{3}$; Patient B: $\left.\mathrm{f}-\mathrm{j}\right)$, and 2D ${ }^{\text {high }} 3 \mathrm{D}^{\text {high }}$ in the proximal LAD (2D-PMR, 1.96; 3Di-PMR, $344 \mathrm{PMR}^{*} \mathrm{~mm}^{3}$; Patient $C: k-o)$. Computed tomography angiography (CTA) images $(a, f, k)$, and axial images $(b, g, l)$, sagittal images $(c, h, m)$, color maps $(d, l, n)$, and 3D region of interests (3D plaque: $\mathrm{e}, \mathrm{j}, \mathrm{n}$ ) on T1w images are shown. Yellow circles indicate percutaneous coronary intervention target lesion sites on CTA. Yellow arrows indicate lesions on T1w imaging corresponding to a lesion on angiography that underwent intervention.

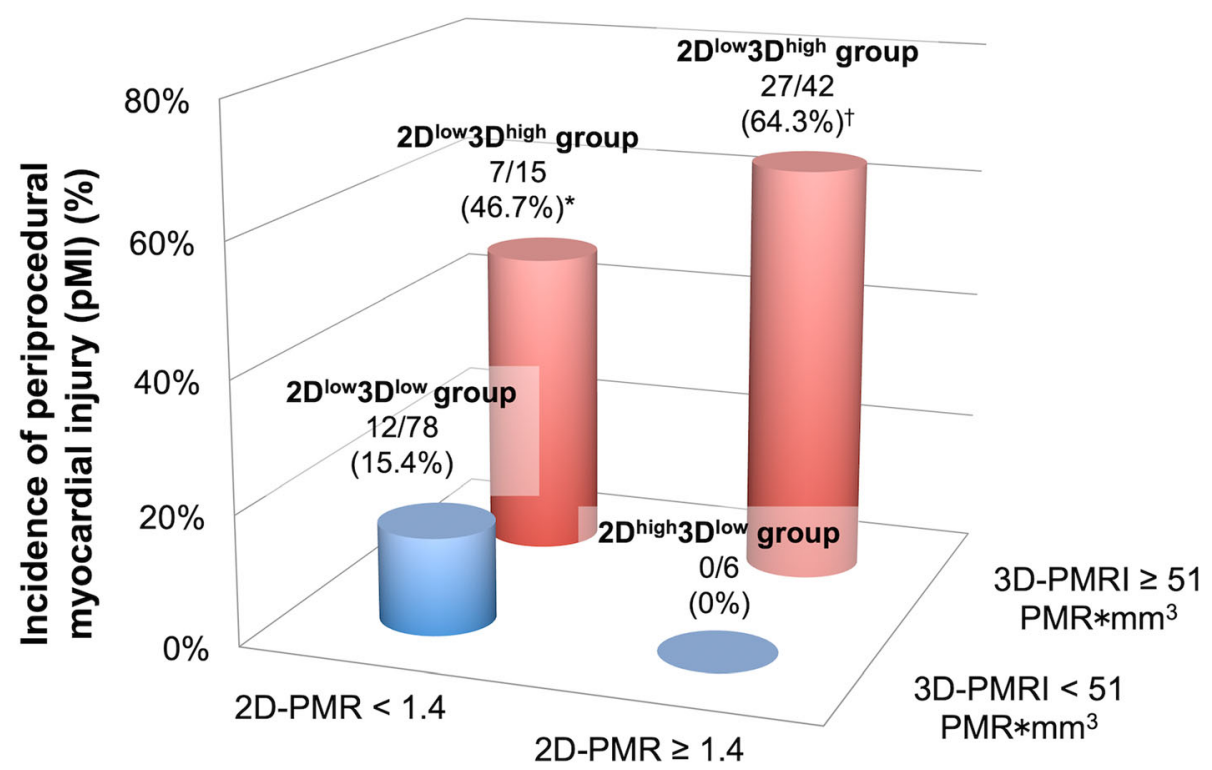

Fig. 5 Incidence of periprocedural myocardial injury (pMI) based on 3Di-PMR and 2D-PMR cutoff values. The red and blue bars represent patients with 3 Di-PMR $\geq 51 \mathrm{PMR}^{*} \mathrm{~mm}^{3}$ and $<51 \mathrm{PMR}^{*} \mathrm{~mm}^{3}$, respectively. $P<0.001$ based on the chi-squared test. $P=0.006 \mathrm{vs}^{*} 2 \mathrm{D}^{\text {high }} 3 \mathrm{D}^{\text {low }} \mathrm{group}^{\dagger} P<$ 0.001 vs. $2 D^{\text {low }} 3 D^{\text {low }}$ group, and $P=0.003$ vs. $2 D^{\text {high }} 3 D^{\text {low }}$ group 
Table 4 Univariable and Multivariable Analyses of Predictors for Periprocedural Myocardial Injury

\begin{tabular}{|c|c|c|c|c|c|c|c|c|c|}
\hline \multirow[t]{2}{*}{ Variable } & \multicolumn{3}{|c|}{ Univariable } & \multicolumn{3}{|c|}{ Multivariable } & \multicolumn{3}{|c|}{ Stepwise } \\
\hline & $\mathrm{OR}$ & $95 \% \mathrm{Cl}$ & $P$ & $\mathrm{OR}$ & $95 \% \mathrm{Cl}$ & $P$ & $\mathrm{OR}$ & $95 \% \mathrm{Cl}$ & $P$ \\
\hline $3 \mathrm{Di}-\mathrm{PMR} \geq 51 \mathrm{PMR}^{*} \mathrm{~mm}^{3}$ & 8.87 & $(3.95-19.9)$ & $<0.001$ & 10.7 & $(3.85-29.7)$ & $<0.001$ & 11.9 & $(4.63-30.4)$ & $<0.001$ \\
\hline Ultrasound attenuation & 3.52 & $(1.41-8.83)$ & 0.007 & 2.71 & $(0.83-8.87)$ & 0.100 & & & \\
\hline Calcification & 1.04 & $(0.41-2.64)$ & 0.935 & 0.86 & $(0.24-3.08)$ & 0.818 & & & \\
\hline Type B2/C lesion & 1.23 & $(0.60-2.53)$ & 0.568 & 0.57 & $(0.22-1.53)$ & 0.266 & & & \\
\hline Positive remodeling & 1.49 & $(0.70-3.19)$ & 0.301 & 0.98 & $(0.37-2.63)$ & 0.969 & & & \\
\hline Side branch & 2.75 & $(1.25-6.04)$ & 0.012 & 5.83 & $(1.86-18.3)$ & 0.003 & 4.14 & $(1.55-11.1)$ & 0.005 \\
\hline Total plaque volume (per 1-mm³ increase) & 1.00 & $(1.00-1.01)$ & 0.170 & 1.00 & $(0.99-1.01)$ & 0.879 & & & \\
\hline
\end{tabular}

3Di-PMR 3-dimensional integral of the plaque-to-myocardium signal intensity ratio, $\mathrm{Cl}$ Confidence interval, $O R$ Odds ratio, $P M R^{*} m m^{3}$ the unit of 3 Di-PMR; defined as the integral of voxel volume multiplied by its PMR value $>1.0$ from a coronary artery

were significant predictors of pMI (odds ratio [OR], 11.9; 95\% CI, 4.63-30.4; $P<0.001$ and OR, 4.14; 95\% CI, 1.5511.1, $P=0.005$; respectively).

Finally, we compared between the 3Di-PMR and coronary CTA derived predictors of pMI previously described to be indicative of high-risk plaque among the 57 patients who underwent CTA (Additional file 1: Tables S1-S2). From ROC analysis, 3Di-PMR also had higher AUC (0.777 [95\% CI, 0.644-0.910]) than CTA-derived indices with significance or marginal significance; CT value $(0.609,[95 \% \mathrm{CI}$, 0.644-0.910]; $P=0.051)$, remodeling index $(0.618$, [95\% CI, 0.461-0.774]; $P=0.051$ ), low attenuation plaque (LAP; 0.566 [95\%CI, 0.427-0.704], $P=0.007$ ), positive remodeling (PR; 0.579 [95\% CI, 0.445-0.713], $P=0.007$ ), spotty calcification $(0.526$ [95\% CI, 0.367-0.666], $P=0.022)$, and LAP + PR (0.651 [95\% CI, 0.501-0.801], $P=0.068)$.

\section{Discussion}

The major finding of this study is that the presence of a coronary HIP with 3Di-PMR $\geq 51 \mathrm{PMR}^{*} \mathrm{~mm}^{3}$ detected by non-contrast $\mathrm{T} 1 \mathrm{w}$ imaging was a significant independent predictor of PCI-related pMI compared to conventional 2D-PMR and other IVUS- or CAG-derived predictors. Thus, noninvasive characterization of coronary plaques based on 3Di-PMR with CMR, which includes the concept of plaque burden extent, is clinically informative for risk stratification of patients with elective PCI.

Although PCI is an important coronary revascularization strategy, especially in patients with $\mathrm{CAD}$, the effect of PCI on clinical outcomes is limited despite the development of contemporary PCI techniques and devices [18]. Thus, reducing the number of complications during PCI may improve the clinical outcomes of PCI. pMI is a complication of PCI whose mechanism is thought to be related to side branch occlusion, atherosclerotic embolism, or both during PCI and may be associated with cardiovascular events after PCI $[2,3]$. The present study shows that novel 3D evaluation of coronary plaques using noncontrast $\mathrm{T} 1 \mathrm{w}$ imaging could facilitate the prediction of pMI after elective PCI. Previous reports have shown that characterizing coronary HIPs with non-contrast T1w imaging is a promising noninvasive method and a novel biomarker for identifying high-risk coronary plaques without any radiation or iodinated contrast exposure [4-7]. Currently, coronary plaques on $\mathrm{T} 1 \mathrm{w}$ imaging are quantified using the signal intensity ratio between plaque and a reference point (i.e., nearby myocardium). This value, 2DPMR, characterizes plaques without taking into account the concept of plaque volume. Matsumoto et al. reported a relationship between qualitative coronary PMR morphology (e.g., intrawall or intraluminal high-intensity signals) visualized with non-contrast $\mathrm{T} 1 \mathrm{w}$ imaging and their clinical severity [19]. In addition, since larger coronary plaque volume was associated with future cardiovascular events $[8,9,20]$, we have developed a novel technique for quantitative 3D plaque analysis with non-contrast $\mathrm{T} 1 \mathrm{w}$ imaging (Figs. 1 and 2). Indeed, as shown in Table 3, the present $3 \mathrm{D}$ coronary plaque assessment produces a more sensitive predictor of pMI after elective PCI than current 2D-PMR and indices derived from IVUS or coronary angiography. It may also be a better predictor of pMI than CTAderived factors (Additional file 1: Tables S1-S2), because CMR was reported to be superior to CTA in terms of characterizing noncalcified, atherosclerotic plaques in an experimental rabbit atherosclerotic model [21], and current 3D plaque assessment may involve both plaque characteristics and their volume. As shown in Figure 5, the prevalence of pMI was higher in patients with $3 \mathrm{Di}$ $\mathrm{PMR} \geq 51 \mathrm{PMR}^{*} \mathrm{~mm}^{3}$ than in those with $3 \mathrm{Di}-\mathrm{PMR}<51$ $\mathrm{PMR}^{*} \mathrm{~mm}^{3}$, irrespective of their 2D-PMR value. Additional file 1: Table S3 also showed that patients with higher 3Di-PMR had positive vessel remodeling compared with patients with lower 3Di-PMR, and patients with higher 2D-PMR had longer attenuation length compared with patients with lower 2D-PMR. Because coronary plaque volume and composition play an important role in pMI after elective PCI, the present 3D evaluation using non-contrast $\mathrm{T} 1 \mathrm{w}$ imaging for coronary atherosclerosis may improve the accuracy of predicting cardiac events. To decrease the incidence of pMI and improve clinical 
outcomes, this noninvasive preoperative evaluation before scheduled PCI might facilitate risk stratification for subgroups at high risk for pMI, who might then benefit from intensive pharmacological approaches (e.g., statins and antiplatelet therapy) and usage of filter devices during elective PCI procedures [22-25].

As shown in Figure 3 and Table 2, total plaque volume and lipid-rich plaque as assessed by IVUS were significantly correlated with 3Di-PMR. In carotid atherosclerosis, HIP atherosclerotic lesions detected by noncontrast T1w imaging consist of complex atheromas with large necrotic cores and intraplaque hemorrhage $[26,27]$. Our previous case report showed that coronary emboli during PCI, detected as a coronary HIP on noncontrast $\mathrm{T} 1 \mathrm{w}$ imaging before $\mathrm{PCI}$, is composed of a large necrotic core with cholesterol crystals and thrombus [10]. Additionally, a histological analysis using formalinfixed post-mortem human hearts, which were imaged at $1.5 \mathrm{~T}$ with $\mathrm{T} 1 \mathrm{w}$ imaging with fat suppression, showed that the majority of coronary HIPs on T1w imaging was reflect intraplaque hemorrhage [28]. Thus, coronary HIPs detected on T1w imaging could include complex atheromas with intraplaque hemorrhage. The precise relationship between plaque burden with intraplaque hemorrhage and pMI during PCI remains unclear. Further studies are needed to clarify the relationship among MRI findings, associated histological and molecular characteristics, and clinical events in coronary atherosclerosis.

\section{Limitations}

Our study has several limitations. The number of patients was small and there might have been selection bias related to how patients were chosen for CMR. Second, the algorithm for $3 \mathrm{D}$ plaque evaluation requires discussion. The Step 1 algorithm, which is based on automated 3D region growing for a coronary plaque with higher signal intensity than nearby myocardium, allowed for 3D quantification of coronary plaques in 128 of 141 patients (91\%). The other 13 patients required additional steps. In particular, the Step 3 algorithm required an increment of the lower segmentation threshold for plaque segmentation until there was no longer expansion beyond the vessel boundaries. These additional steps could hamper this assessment in more complex lesions and have little impact on predicting clinical outcomes. However, there is currently no method to quantify plaque morphology on T1w imaging. Thus, further studies to develop plugin software or other algorithms for plaque segmentation are needed for 3D plaque quantification with CMR in coronary atherosclerosis. Third, we did not perform systemic analyses to compare the findings between coronary CMR and CTA, which could also predict pMI.

\section{Conclusion}

Three-dimensional assessment of coronary plaques reflecting both plaque volume and composition facilitates more accurate risk stratification and prediction of pMI after elective PCI in stable CAD patients.

\section{Supplementary information}

Supplementary information accompanies this paper at https://doi.org/10. 1186/s12968-019-0588-6.

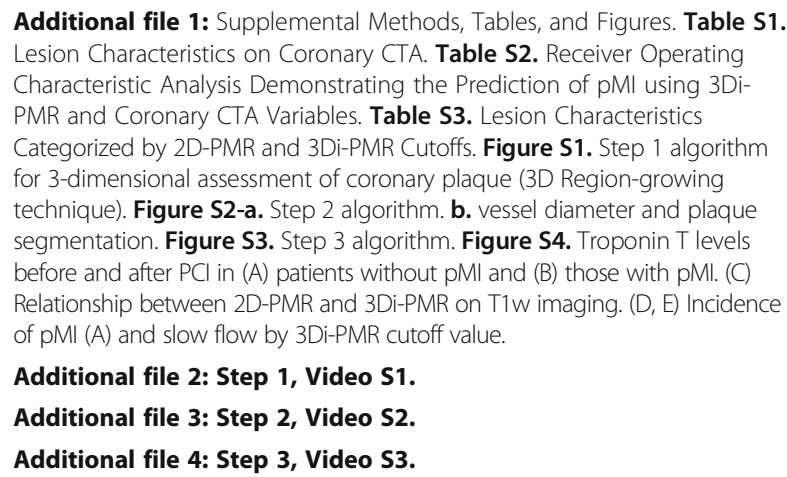

\section{Abbreviations}

2D: 2-dimensional; 3D: 3-dimensional; 3Di: 3-dimensional integral; CAD: Coronary artery disease; Cl: Confidence interval; CMR: Cardiovascular magnetic resonance; CTA: Coronary computed tomography angiography; ECG: Electrocardiogram; HIP: High intensity plaque; hs-TnT: High-sensitivity troponin T; IQR: Interquartile range; IVUS: Intravascular ultrasound; OR: Odds ratio; PCl: Percutaneous coronary intervention; pMl: Periprocedural myocardial injury; PMR: Plaque-to-myocardial signal intensity ratio; ROI: Region of interest; T1w: T1-weighted; TIMI: Thrombolysis In Myocardial Infarction

\section{Acknowledgements}

We thank Saki Yamamoto, Yasutaka Nakahira, and Sakura Amimoto (CHURUN Company, Osaka, Japan) for preparing the movie files in this manuscript.

\section{Authors' contributions}

YA and TNo conceived and designed this study. HH, YA, TNo, and SY implemented the study protocol, performed data analysis, and wrote the manuscript. YM and YKo set up the CMR sequence. YM and KNa performed the CMR image analysis. YKo, TH, AS, and TK supervised the CMR image analysis. KNa and AK performed the CT analysis. $\mathrm{HH}$. . MN and KNi performed the statistical analysis. TNa, FM, Cl, KK, TF, and SY critically revised the manuscript. All authors read and approved the final manuscript.

\section{Funding \\ This study was supported in part by Grants-in-Aid for Science Research (KAKENHI grant 18 K15872 to Dr. Hosoda; 17 K09566 to Dr. Asaumi), grants from the Japan Agency for Medical Research and Development (grant num- ber 17ek0210085h0001 to Dr. Noguchi), SENSIN IYAKU Foundation (to Dr. Asaumi), Daiwa Securities Health Foundation (to Dr. Asaumi), and Smoking Research Foundation (to Dr. Yasuda).}

\section{Availability of data and materials}

The datasets used and/or analyzed during the current study are available from the corresponding author on reasonable request.

\section{Ethics approval and consent to participate}

This study was approved by the Institutional Review Board at National Cerebral and Cardiovascular Center (M26-037-2). All subjects provided informed consent for research participation.

Consent for publication

Not applicable, non-identifiable data only included. 


\section{Competing interests}

Yoshiaki Komori is employee of Siemens Japan KK. Other authors declare that they have no competing interests.

\section{Author details \\ ${ }^{1}$ Department of Cardiovascular Medicine, National Cerebral and Cardiovascular Center, 6-1 Kishibe-Shimmachi, Suita 564-8565, Japan. ${ }^{2}$ Department of Advanced Cardiovascular Medicine, Graduate School of Medical Sciences, Kumamoto University, Kumamoto, Japan. ${ }^{3}$ Department of Radiology, National Cerebral and Cardiovascular Center, Suita, Japan. ${ }^{4}$ Department of Preventative Cardiology, National Cerebral and Cardiovascular Center, Suita, Japan. ${ }^{5}$ Department of Research and Collaboration, Siemens Japan KK, Tokyo, Japan. ${ }^{6}$ Department of Cardiovascular Medicine, University of Tsukuba, Tsukuba, Japan. ${ }^{7}$ Cardiovascular Center, Shin-Koga Hospital, Kurume, Japan.}

\section{Received: 24 May 2019 Accepted: 21 November 2019}

Published online: 16 January 2020

\section{References}

1. Prasad A, Singh M, Lerman A, Lennon RJ, Holmes DR Jr, Rihal CS. Isolated elevation in troponin $T$ after percutaneous coronary intervention is associated with higher long-term mortality. J Am Coll Cardiol. 2006;48:1765-70.

2. Prasad A, Herrmann J. Myocardial infarction due to percutaneous coronary intervention. N Engl J Med. 2011;364:453-64.

3. Zeitouni M, Silvain J, Guedeney P, Kerneis M, Yan Y, Overtchouk $P$, Barthelemy O, Hauguel-Moreau M, Choussat R, Helft G, Le Feuvre C, Collet JP, Montalescot G, Group AS. Periprocedural myocardial infarction and injury in elective coronary stenting. Eur Heart J. 2018;39:1100-9.

4. Kawasaki T, Koga S, Koga N, Noguchi T, Tanaka H, Koga H, Serikawa T, Orita Y, Ikeda S, Mito T, Goto Y, Shintani Y, Tanaka A, Fukuyama T. Characterization of hyperintense plaque with noncontrast $\mathrm{T}(1)$-weighted cardiac magnetic resonance coronary plaque imaging: comparison with multislice computed tomography and intravascular ultrasound. JACC Cardiovasc Imaging. 2009;2:720-8.

5. Noguchi T, Kawasaki T, Tanaka A, Yasuda S, Goto Y, Ishihara M, Nishimura K, Miyamoto $Y$, Node K, Koga N. High-intensity signals in coronary plaques on noncontrast t1-weighted magnetic resonance imaging as a novel determinant of coronary events. J Am Coll Cardiol. 2014;63:989-99.

6. Asaumi Y, Noguchi T, Morita Y, Fujiwara R, Kanaya T, Matsuyama TA, Kawasaki T, Fujino M, Yamane T, Nagai T, Miyagi T, Ishibashi-Ueda H, Higashi M, Komori Y, Ishihara M, Ogawa H, Yasuda S. High-intensity plaques on noncontrast T1-weighted imaging as a predictor of Periprocedural myocardial injury. JACC CardiovasC Imaging. 2015;8:741-3.

7. Hoshi T, Sato A, Akiyama D, Hiraya D, Sakai S, Shindo M, Mori K, Minami M, Aonuma K. Coronary high-intensity plaque on T1-weighted magnetic resonance imaging and its association with myocardial injury after percutaneous coronary intervention. Eur Heart J. 2015;36:1913-22.

8. Virmani R, Burke AP, Kolodgie FD, Farb A. Vulnerable plaque: the pathology of unstable coronary lesions. J Interv Cardiol. 2002;15:439-46.

9. Uetani T, Amano T, Ando H, Yokoi K, Arai K, Kato M, Marui N, Nanki M, Matsubara T, Ishii H, Izawa H, Murohara T. The correlation between lipid volume in the target lesion, measured by integrated backscatter intravascular ultrasound, and post-procedural myocardial infarction in patients with elective stent implantation. Eur Heart J. 2008;29:1714-20.

10. Asaumi Y, Noguchi T, Morita Y, Matsuyama TA, Otsuka F, Fujiwara R, Kanaya T, Nagai T, Higashi M, Kusano K, Anzai T, Ishibashi-Ueda H, Ogawa H, Yasuda S. Non-contrast T1-weighted magnetic resonance imaging at 3.0 Tesla in a patient undergoing elective percutaneous coronary intervention - clinical and pathological significance of high-intensity plaque. Circ J. 2015;79:218-20.

11. Kanaya T, Noguchi T, Otsuka F, Asaumi Y, Kataoka Y, Morita Y, Miura H, Nakao K, Fujino M, Kawasaki T, Nishimura K, Inoue T, Narula J, Yasuda S. Optical coherence tomography-verified morphological correlates of high-intensity coronary plaques on non-contrast T1-weighted magnetic resonance imaging in patients with stable coronary artery disease. Eur Heart J Cardiovasc Imaging. 2019;20:75-83.

12. Ibrahim T, Makowski MR, Jankauskas A, Maintz D, Karch M, Schachoff S, Manning WJ, Schomig A, Schwaiger M, Botnar RM. Serial contrast-enhanced cardiac magnetic resonance imaging demonstrates regression of hyperenhancement within the coronary artery wall in patients after acute myocardial infarction. JACC Cardiovasc Imaging. 2009;2:580-8.

13. Neumann FJ, Sousa-Uva M, Ahlsson A, Alfonso F, Banning AP, Benedetto U, Byrne RA, Collet JP, Falk V, Head SJ, Juni P, Kastrati A, Koller A, Kristensen SD,
Niebauer J, Richter DJ, Seferovic PM, Sibbing D, Stefanini GG, Windecker S, Yadav R, Zembala MO, Group ESCSD. 2018 ESC/EACTS guidelines on myocardial revascularization. Eur Heart J. 2019;40:87-165.

14. Heuberger J, Rosset A. OsiriX User Manual. 5th ed; 2017.

15. Yamada M, Jinzaki M, Kuribayashi S, Sato K, Tanami Y, Yun S, Sasaki K. Evaluation of coronary artery lumen diameter with 16-slice multidetectorrow computed tomography. Circ J. 2005;69:1230-2.

16. Miyagi T, Asaumi Y, Nishimura K, Nakashima T, Sakamoto H, Nakao K, Kanaya T, Nagai T, Shimabukuro Y, Miyamoto Y, Fujita T, Kusano K, Anzai T, Kobayashi J, Noguchi T, Ogawa H, Yasuda S. Validation of the coronary artery bypass graft SYNTAX score (synergy between percutaneous coronary intervention with Taxus) as a prognostic marker for patients with previous coronary artery bypass graft surgery after percutaneous coronary intervention. Circ Cardiovasc Interv. 2016;9:e003459.

17. Endo M, Hibi K, Shimizu T, Komura N, Kusama I, Otsuka F, Mitsuhashi T, Iwahashi N, Okuda J, Tsukahara K, Kosuge M, Ebina T, Umemura S, Kimura K. Impact of ultrasound attenuation and plaque rupture as detected by intravascular ultrasound on the incidence of no-reflow phenomenon after percutaneous coronary intervention in ST-segment elevation myocardial infarction. JACC Cardiovasc Interv. 2010;3:540-9.

18. Boden WE, O'Rourke RA, Teo KK, Hartigan PM, Maron DJ, Kostuk WJ, Knudtson M, Dada M, Casperson P, Harris CL, Chaitman BR, Shaw L, Gosselin G, Nawaz S, Title LM, Gau G, Blaustein AS, Booth DC, Bates ER, Spertus JA, Berman DS, Mancini GB, Weintraub WS, Group CTR. Optimal medical therapy with or without PCI for stable coronary disease. N Engl J Med. 2007;356:1503-16.

19. Matsumoto K, Ehara S, Hasegawa T, Sakaguchi M, Otsuka K, Yoshikawa J, Shimada K. Localization of coronary high-intensity signals on T1-weighted MR imaging: relation to plaque morphology and clinical severity of angina pectoris. JACC Cardiovasc Imaging. 2015;8:1143-52.

20. Stone GW, Maehara A, Lansky AJ, de Bruyne B, Cristea E, Mintz GS, Mehran R, McPherson J, Farhat N, Marso SP, Parise H, Templin B, White R, Zhang Z, Serruys PW, Investigators P. A prospective natural-history study of coronary atherosclerosis. N Engl J Med. 2011;364:226-35.

21. Viles-Gonzalez JF, Poon M, Sanz J, Rius T, Nikolaou K, Fayad ZA, Fuster V, Badimon JJ. In vivo 16-slice, multidetector-row computed tomography for the assessment of experimental atherosclerosis: comparison with magnetic resonance imaging and histopathology. Circulation. 2004;110:1467-72.

22. Di Sciascio G, Patti G, Pasceri V, Gaspardone A, Colonna G, Montinaro A. Efficacy of atorvastatin reload in patients on chronic statin therapy undergoing percutaneous coronary intervention: results of the ARMYDARECAPTURE (atorvastatin for reduction of myocardial damage during angioplasty) randomized trial. J Am Coll Cardiol. 2009;54:558-65.

23. Patti G, Colonna G, Pasceri V, Pepe LL, Montinaro A, Di Sciascio G. Randomized trial of high loading dose of clopidogrel for reduction of periprocedural myocardial infarction in patients undergoing coronary intervention: results from the ARMYDA-2 (antiplatelet therapy for reduction of MYocardial damage during angioplasty) study. Circulation. 2005;111:2099-106.

24. Basili S, Tanzilli G, Raparelli V, Calvieri C, Pignatelli P, Carnevale R, Dominici M, Placanica A, Arrivi A, Farcomeni A, Barilla F, Mangieri E, Violi F. Aspirin reload before elective percutaneous coronary intervention: impact on serum thromboxane b2 and myocardial reperfusion indexes. Circ Cardiovasc Interv. 2014;7:577-84.

25. Claessen BE, Maehara A, Fahy M, Xu K, Stone GW, Mintz GS. Plaque composition by intravascular ultrasound and distal embolization after percutaneous coronary intervention. JACC Cardiovasc Imaging. 2012;5:S111-8.

26. Moody AR, Murphy RE, Morgan PS, Martel AL, Delay GS, Allder S, MacSweeney ST, Tennant WG, Gladman J, Lowe J, Hunt BJ. Characterization of complicated carotid plaque with magnetic resonance direct thrombus imaging in patients with cerebral ischemia. Circulation. 2003;107:3047-52.

27. Yuan C, Mitsumori LM, Ferguson MS, Polissar NL, Echelard D, Ortiz G, Small R, Davies JW, Kerwin WS, Hatsukami TS. In vivo accuracy of multispectral magnetic resonance imaging for identifying lipid-rich necrotic cores and intraplaque hemorrhage in advanced human carotid plaques. Circulation. 2001;104:2051-6.

28. Kuroiwa Y, Uchida A, Yamashita A, Miyati T, Maekawa K, Gi T, Noguchi T, Yasuda S, Imamura T, Asada Y. Coronary high-signal-intensity plaques on T1-weighted magnetic resonance imaging reflect intraplaque hemorrhage. Cardiovasc Pathol. 2019;40:24-31.

\section{Publisher's Note}

Springer Nature remains neutral with regard to jurisdictional claims in published maps and institutional affiliations. 